\title{
Hungerstreik und Zwangsernährung
}

\section{Markus Müller ${ }^{a}$, \\ Christoph Jenni $^{b}$}

a Prof. Dr. iur., Ordinarius für Staats- und Verwaltungsrecht an der Universität Bern

b Dr. iur., Assistent am Institut für öffentliches Recht der Universität Bern

Ein Kommentar der FMH findet sich auf Seite 287

\footnotetext{
* Die Literaturangaben finden sich online unter www.saez.ch $\rightarrow$ Aktuelle Nummer oder

$\rightarrow$ Archiv $\rightarrow 2011 \rightarrow 8$.
}

Korrespondenz:

Prof. Dr. iur. Markus Müller Universität Bern

Institut für öffentliches Recht Schanzeneckstrasse 1

Postfach 8573

CH-3001 Bern

Tel. 0316314594

markus.mueller@oefre.unibe.ch

www.oefre.unibe.ch
Der Walliser Hanfbauer Bernhard Rappaz bewegte mit seinem lang andauernden Hungerstreik die Gemüter und löste zahlreiche Kontroversen aus. Nach 120 Tagen hat er seinen Protest zwar abgebrochen, aber die offenen Fragen zum Thema Hungerstreik im Strafvollzug bleiben. Die Abwesenheit eines akuten Falles gibt nun die nötige Ruhe, um die ethisch, juristisch und politisch umstrittenen Punkte weiter zu diskutieren. In diesem Sinn möchte der vorliegende Beitrag die interdisziplinäre Diskussion weiterführen. Als Anknüpfungspunkt dient die in der SÄZ 39/2010 publizierte Verlautbarung der Ärzteschaft [1]*, der anhand dreier Fragestellungen einige juristische Sicht- und Denkweisen entgegengesetzt werden sollen: 1) Worin liegt die Herausforderung eines Hungerstreiks für Recht und Medizin? 2) Bietet die Zwangsernährung einen juristisch gangbaren Ausweg? 3) Was sind die Rechte und Pflichten der Gefängnisärzte?

\section{Hungerstreik als Herausforderung für Recht und Medizin}

Tritt ein Strafgefangener in den Hungerstreik, um damit gegen Haftumstände zu protestieren oder gar die Haftentlassung zu fordern [2], führt dies zu einer Kollision hochwertiger Interessen seitens des Streikenden wie auch des Staates. Oberflächlich betrachtet, kreist der Konflikt um das Selbstbestimmungsrecht des Gefangenen einerseits und den staatlichen Strafanspruch andererseits. Doch der simple Schein vom bipolaren Spannungsfeld trügt, denn an beiden Polen sammeln sich zuweilen auch gegenläufige Interessen. So ist nicht nur die rechtsgleiche und geordnete Durchsetzung des Strafanspruchs ein Anliegen des Staates, sondern auch die möglichst weitgehende Wahrung der Grund- und Menschenrechte der Strafgefangenen wie auch seiner Bediensteten (z.B. der Gefängnisärzte). Diese multipolare Konfliktlage bringt alle Beteiligten unweigerlich in Bedrängnis - je länger der Hungerstreik dauert, umso mehr.

Ein Ausweg ist weder für den Streikenden selber noch für die staatliche Vollzugsbehörde einfach zu finden. Im Idealfall gelingt es, den Gefangenen zur Aufgabe seines Protestes zu bewegen, etwa durch aufklärende Gespräche oder «Verführen» mittels köstlicher Speisen [3]. Was aber, wenn er sich nicht von seinem Unterfangen abbringen lässt? Bleibt auch die Vollzugsbehörde unnachgiebig, verbleibt nur die Wahl zwischen «Pest und Cholera»: Entweder stirbt der Gefangene den Hungertod oder der Staat führt eine Zwangsernährung durch. Darüber, was zu tun ist, lässt sich nunmehr trefflich streiten. Allerdings stehen Mediziner wie auch Juristen regelmässig unter Ent- scheidungsdruck. Insoweit befinden sie sich in vergleichbaren Rollen, was die interdisziplinäre Verständigung in gewisser Hinsicht erleichtert.

\section{Zwangsernährung als Ausweg?}

Einschränkung von Grund- und Menschenrechten Eine zwangsweise durchgeführte künstliche Ernährung (z.B. mittels Magensonde oder Infusion) stellt einen schwerwiegenden Eingriff in elementare Grund- und Menschenrechtspositionen des Gefangenen dar. Zuallererst ist das Recht auf Selbstbestimmung betroffen. Dieses erlaubt grundsätzlich auch einem Strafgefangenen, nach freiem Willen über den eigenen Körper und das eigene Leben zu verfügen. Weiter schränkt die Zwangsernährung das Recht auf psychische und körperliche Integrität, die Bewegungsfreiheit sowie das Recht auf freie Meinungsäusserung ein.

Nicht betroffen ist hingegen das Folterverbot bzw. das Verbot unmenschlicher oder erniedrigender Behandlung, jedenfalls solange die Zwangsernährung dem Schutz des Lebens dient und in würdewahrender Weise erfolgt. Dies haben - entgegen der Darstellung auf Seite 1524 in der SÄZ 39/2010 - sowohl der Europäische Gerichtshof für Menschenrechte wie auch das Bundesgericht festgehalten [4].

Der Umstand, dass die Zwangsernährung diverse Grundrechtspositionen einschränkt, bedeutet noch nicht deren Verfassungswidrigkeit. Jeder Eingriff kann nämlich gerechtfertigt werden, wenn er in einer hinreichend bestimmten gesetzlichen Grundlage vorgesehen ist, einem öffentlichen Interesse dient und verhältnismässig ausgestaltet ist.

\section{Gesetzliche Grundlage}

Die Anforderungen an die gesetzliche Grundlage sind bei der Zwangsernährung - einem schweren Grundrechtseingriff -, besonders hoch. Gefordert ist eine formell-gesetzliche Vorschrift, die die Grundzüge und Rahmenbedingungen der Massnahme hinreichend klar und bestimmt umschreibt. Eine solche Vorschrift findet sich z. B. im Straf- und Massnahmenvollzugsgesetz des Kantons Bern. Dort wird eine Zwangsernährung erlaubt, wenn eine schwerwiegende Gefahr für den Hungerstreikenden besteht und nicht «von einer freien Willensbestimmung der betroffenen Person ausgegangen werden» muss [5]. In den meisten anderen Kantonen fehlt jedoch eine ähnliche gesetzliche Grundlage.

Wo eine gesetzliche Grundlage fehlt, können die kantonalen Vollzugsbehörden zwar ausnahmsweise, gestützt auf die sog. polizeiliche Generalklausel, han- 
deln, allerdings nur unter restriktiven Bedingungen. Nach Auffassung des höchsten Gerichts waren diese im Fall Rappaz gegeben [6].

Spätestens der Fall Rappaz hat deutlich gemacht, dass die Frage des Umgangs mit hungerstreikenden Strafgefangenen dringend einer Regelung bedarf, sei es auf Stufe Bund [7] oder im Rahmen der drei kantonalen Strafvollzugskonkordate. $\mathrm{Zu}$ beachten gilt allerdings, dass eine explizite Regelung der Zwangsernährung auch kontraproduktiv sein kann: Weiss der Hungerstreikende von Beginn an, dass ihn die Behörden mit Sicherheit künstlich ernähren werden, so kann er alles riskieren. Dabei gewinnt er doppelte Aufmerksamkeit als scheinbar zu allem bereiter Kämpfer für die eigene Sache sowie als Märtyrer, dessen Widerstand gegen die Obrigkeit mit Gewalt gebrochen wird. Kann sich der Streikende dagegen nicht

\section{In einer Güterabwägung wird sich die einmalige Zwangsernährung tendenziell als verhältnismässige Massnahme erweisen.}

darauf verlassen, am Leben erhalten zu werden, muss er sich zwangsläufig selber schonen und seinen Streik «massvoll» umsetzen. Eine zukünftige gesetzliche Regelung muss diesem Erpressungspotential Rechnung tragen.

\section{Öffentliches Interesse und Verhältnismässigkeit}

Unabhängig von der Eingriffsgrundlage muss eine Zwangsernährung auch in einem öffentlichen Interesse liegen. Öffentliche Interessen sind im vorliegenden Zusammenhang problemlos zu finden: der Schutz des Lebens und der Gesundheit von Strafgefangenen, die Durchsetzung des staatlichen Strafanspruchs, ein geordneter und funktionierender Strafvollzug, die Gleichbehandlung der Strafgefangenen, die Glaubwürdigkeit der Justiz, die Verhinderung von Nachahmern usw.

Schwieriger zu beantworten ist die Frage, ob ein Eingriff von der Intensität der Zwangsernährung verhältnismässig ist; das heisst, ob er die angerufenen öffentlichen Interessen aufzuwiegen vermag. Diese Frage lässt sich nur aufgrund einer Abwägung der gegenüberstehenden privaten und öffentlichen Interessen beurteilen:

- Staatliche Fürsorgepflicht: Der Strafvollzug schafft für den Inhaftierten ein besonderes Rechtsverhältnis. Dieses ist dadurch gekennzeichnet, dass der Staat auf der einen Seite massiv in seine Freiheitsrechte eingreifen darf, auf der anderen Seite aber auch zu erhöhter Fürsorge verpflichtet ist. Die Fürsorge umfasst einerseits den Schutz elementarer Grundrechtspositionen (insbesondere den Schutz des Lebens als Voraussetzung jeder Grund- rechtswahrnehmung), berechtigt andererseits aber auch zur Beschränkung des Selbstbestimmungsrechts. Sowohl das staatliche Eingriffsrecht als auch die staatliche Fürsorgepflicht gestalten sich somit gegenüber einem Gefangenen deutlich intensiver als gegenüber einem in Freiheit lebenden Menschen.

- Zweck des Hungerstreiks: Ein Hungerstreik, der sich gegen den Vollzug einer rechtmässig ausgesprochenen Strafe richtet und insoweit einen widerrechtlichen Zweck verfolgt, erscheint weniger gewichtig als ein Hungerstreik, der grundsätzlich legitime Anliegen zum Ziel hat (z. B. Verbesserung der Haftbedingungen [8]). Das Selbstbestimmungsrecht des Gefangenen erlaubt diesem mit anderen Worten nicht, sein Leben zu riskieren, um damit den Rechtsstaat unter Druck zu setzen [9]. Ein streikender Strafgefangener kann folglich sein Selbstbestimmungsrecht nur in dem Umfang anrufen, als es sich mit den Zwecken des Strafvollzugs verträgt.

- Sterbewillen: Der Sterbewillen eines Gefangenen ist grundsätzlich ernst zu nehmen; vorausgesetzt, ein solcher liegt tatsächlich vor. Gerade im Kontext eines Hungerstreiks ist dieser Sterbewille aber häufig alles andere als klar: Will der Streikende tatsächlich sterben oder will er nicht vielmehr unter verbesserten Umständen leben? Der geäusserte Sterbewille entpuppt sich dann als blosse Inkaufnahme eines gewissen Sterberisikos, dem in der Abwägung nicht allzu viel Gewicht beigemessen werden kann.

- Patientenverfügungen: Patientenverfügungen des Inhalts, keine lebenserhaltenden Massnahmen zu tolerieren, sind im Kontext des Hungerstreiks häufig. Sie dienen dem Streikenden, die Ernsthaftigkeit und Zielstrebigkeit seines Unterfangens zu unterstreichen und den Druck dadurch zu erhöhen. Einmal unabhängig von der bereits angesprochenen Vagheit des Sterbewillens, ist der Geltungsanspruch solcher Patientenverfügungen auch aus anderen Gründen zu relativieren: Die Zurechnungsfähigkeit des «Verfügenden» mag zu Beginn des Streiks noch bestanden haben; sie schwindet aber über die folgenden Wochen permanent dahin. Zwischen dem Eintritt der Unzurechnungsfähigkeit und der Bewusstlosigkeit können dann nochmals mehrere Wochen verstreichen [10]. Ein zu Beginn geäusserter Verzicht auf lebenserhaltende Massnahmen kann in dieser Periode kaum mehr mit der nötigen Einsichtsfähigkeit überdacht und bestätigt bzw. widerrufen werden. Aufgestaute Wut- und Verzweiflungsgefühle, aber auch die zunehmende Angst vor einem Gesichtsverlust werden gegebenenfalls vom nunmehr vernebelten «Restverstand» überbewertet und können den Ausweg über den freiwilligen Streikabbruch versperren [11]. Wer kann vor diesem Hintergrund noch von einer «klaren Willens- 
kundgabe», von einer «gültigen Patientenverfügung» sprechen? Und wer kann die Verantwortung für das lebensgefährliche «Laufenlassen» des Geschehens übernehmen?

- Zwangsernährung als Ultima ratio: Die Zwangsernährung ist grundsätzlich geeignet, den Hungertod abzuwenden, auch wenn ihre praktische Durchführung aus medizinischer Sicht durchaus mit Komplikationsrisiken behaftet ist. Angesichts dieser Risiken darf eine Realimentierung nur als Ultima ratio zur Anwendung gelangen. Denn solange die Gesundheit nicht schwerwiegend bedroht ist, bestehen mildere Eingriffsmittel. Mit fortschreitender Schwächung bis hin zum Koma reduzieren sich dann aber die möglichen Alternativen, und es bleibt nur die Zwangsernährung.

In einer Güterabwägung, die all diesen Aspekten Rechnung trägt, wird sich die Zwangsernährung tendenziell als verhältnismässige Massnahme erweisen. Zumindest einmal sollte der Streikende in den physischen und psychischen Zustand zurückversetzt werden, der es ihm erlaubt, seine Situation nochmals bei vollem Bewusstsein zu überdenken. Entscheidet er sich dann - kaum erstarkt - aufs neue für die Wiederaufnahme des Hungerstreiks, endet der staatliche Schutz- und Fürsorgeauftrag. Eine fortdauernde Zwangsernährung wäre weder dem Streikenden noch der Vollzugsbehörde bzw. dem Gefängnisarzt zumutbar und folglich als unverhältnismässig abzulehnen.

\section{Rechte und Pflichten der Gefängnisärzte}

\section{Gefängnisärzte zwischen Profession und Hierarchie}

Eine Zwangsernährung kann nur von Ärzten durchgeführt werden. Es handelt sich dabei um einen anspruchsvollen und risikobehafteten Eingriff. Dieser wird von der Ärzteschaft allerdings mehrheitlich ab-

\section{Eine fortdauernde Zwangsernährung wäre weder dem Streikenden noch dem Gefängnisarzt zumutbar und folglich als unverhältnismässig abzulehnen.}

gelehnt, sofern der Streikende sich (in einer Patientenverfügung) gegen jedwede lebenserhaltende Intervention ausgesprochen hat. Die Zwangsernährung greife - so die Ansicht der Ärzte - in unzulässiger Weise in das Selbstbestimmungsrecht des Gefangenen ein, was einem fundamentalen Verstoss gegen die ärztliche Berufsethik gleichkomme [12]. Auch die Richtlinien der SAMW über die Ausübung der ärztlichen Tätigkeit bei inhaftierten Personen fordern den Gefängnisarzt auf, das Selbstbestimmungsrecht über den Lebensschutz zu stellen. Dabei geht es der Ärzteschaft zweifelsohne in erster Linie um die Rechte ihrer Patienten. Aber geht es nicht auch um eine
Machtfrage und um die Furcht vor einer «Instrumentalisierung» durch die Justiz [13]?

Die ärztliche Auffassung kann jedenfalls aus rechtswissenschaftlicher Sicht nicht unkommentiert bleiben. Es ist das gute Recht jeder Profession, sich (ethische) Standesregeln zu geben. Der Geltungsbereich dieser (privaten Verbands-)Regeln ist aber auf das privatärztliche Handeln beschränkt. Gefängnisärzte übernehmen jedoch eine staatliche Aufgabe, weshalb ihre Tätigkeit als amtlich zu qualifizieren ist. Im Rahmen der Erfüllung dieser amtlichen Aufgabe muss sich der Arzt in das hierarchisch geprägte Verwaltungsgefüge eingliedern und primär das zwingende staatliche Recht beachten; den ärztlichen Standesregeln kommt hierbei nur untergeordnete Bedeutung zu [14].

Ein Dienstbefehl der übergeordneten Stelle, der vom Gefängnisarzt verlangt, einen hungerstreikenden Gefangenen zwangsweise zu ernähren, ist daher grundsätzlich zu befolgen. Wird der Dienstbefehl gar durch einen höchstrichterlichen Rechtsspruch (indirekt) bestätigt, so geschehen im Fall Rappaz, gilt dies umso mehr [15]. Das Bundesgericht hat hier verschiedentlich zum Ausdruck gebracht, dass den Staat für seine Gefangenen eine Fürsorgepflicht trifft, die den Schutz des Lebens höher gewichtet als den Schutz des Selbstbestimmungsrechts.

Ob dieser Richterspruch klug, überzeugend oder gar medizinethisch richtig ist, mag den Gefängnisarzt als Berufsmann kümmern, als staatlicher Bediensteter darf es ihn in letzter Konsequenz jedoch nicht interessieren. Er hat mitzuhelfen, das demokratisch legitimierte Recht konsequent zu vollziehen. Weigert er sich, drohen ihm disziplinar- oder gar strafrechtliche Konsequenzen [16]. Das Recht und die darauf abgestützten rechtskräftigen Entscheide können nicht von Fall zu Fall, je nach ethischer Grundauffassung des betroffenen Bediensteten, immer wieder neu zur Diskussion gestellt werden. Grundlegende Kritik am staatlichen Recht ist im Rahmen des Gesetzgebungsprozesses einzubringen. Kann sich ein Gefängnisarzt mit diesen rechtsstaatlichen Spielregeln nicht einverstanden erklären, muss er sich überlegen, den öffentlichen Dienst zu quittieren.

\section{Gewissensfreiheit des Arztes}

Ein Gefängnisarzt kann sich zwar grundsätzlich auf seine Gewissensfreiheit berufen (Art. 15 Abs. 1 BV), doch muss er Einschränkungen gewärtigen, sofern seine amtliche Funktion solche erfordert und er bei Amtsantritt mit ihnen rechnen musste. Beides dürfte für den Gefängnisarzt in Bezug auf die Durchführung einer Zwangsernährung der Fall sein: Zunächst gehört der medizinisch-therapeutische Umgang mit hungerstreikenden Gefangenen zum Gefängnisalltag [17]. Insofern ist es vorhersehbar, dass die Durchführung einer Zwangsernährung auf den Gefängnisarzt zukommen könnte. Dies umso mehr, als er damit in seiner Kernkompetenz angesprochen wird: nämlich Leben zu schützen und zu erhalten - immerhin der Grundauftrag ärztlichen Handelns [18]. Selbst ausser- 


\section{Kommentar von Hanspeter Kuhn, Fürsprecher und stv. Generalsekretär der FMH}

Der nun abgebrochene Hungerstreik von Hanfbauer Rappaz hat über Monate die Gemüter in der Romandie bewegt. Die Staats- und Verwaltungsrechtler Prof. Markus Müller und Dr. Christoph Jenni haben dargelegt, weshalb sie die Auffassung vertreten, der Staat sei berechtigt und verpflichtet, wenigstens einmalig die Zwangsernährung anzuordnen, und weshalb der Gefängnisarzt diese Anordnung durchführen müsse. Ihre Argumente verdienen eine kritische Lektüre. Der Konflikt zwischen der Achtung des Selbstbestimmungsrechts und anderen betroffenen Grundrechten einerseits und den staatlichen Schutzpflichten anderseits bedingt eine Interessenabwägung. Deshalb lassen sich im Ergebnis verschiedene Lösungen rechtlich vertreten [1], und es muss kein Konflikt zwischen Recht und Ethik entstehen.

Persönlich vertrete ich die Meinung, eine Zwangsernährung wäre falsch gewesen, weil ich eine Instrumentalisierung der Medizin befürchte [2]. Die Prof. Olivier Guillod und Dominique Sprumont [3] haben uns daran erinnert, dass der europäische Menschenrechtsgerichtshof in Strassburg die Zwangsernährung ohne medizinische Notwendigkeit auch schon als von der EMRK verbotene Folter eingestuft hatte [4] und umgekehrt einen Staat nicht verurteilte, der keine Zwangsernährung angeordnet hatte $[1,5]$.

1 Vgl. auch die im Ergebnis offene Zwischenbilanz von Claudia Schoch, «Im Dilemma zwischen Legalität und Legitimität», NZZ vom 20.11.2010: «Heute sind wir zögerlicher darin, wie viel Fürsorge der Staat einem Gefangenen angedeihen zu lassen hat und wie schwer er gegen dessen Willen zu seinem Wohl in die persönliche Integrität eingreifen darf.»

2 Unsere Gesetzgebung wurde in vielen Punkten repressiver, denken wir nur an die Verschärfungen der letzten Jahre des Asylrechts, der Invalidenversicherung und des Strafrechts. In anderen Ländern wird Folter wieder salonfähig - und typischerweise muss dabei die Medizin sicherstellen, dass der Gefolterte nicht stirbt. Mir graut vor der Idee, dass in der Schweiz ein Mensch, dem im wesentlichen der Anbau grosser Mengen von Hanf vorgeworfen wurde, durch den Gefängnisarzt gefesselt und mit einem Tubus zwangsernährt werden soll.

3 Guillod O, Sprumont D. Les contradictions du Tribunal fédéral face au jeûne de protestation. In: Jusletter 8.11.2010.

4 Guillod O, Sprumont D, op.cit., [Rz 29] Le Tribunal fédéral aurait pu ajouter que la Cour européenne des droits de l'homme a jugé à au moins deux reprises que, en l'absence de nécessité médicale, l'alimentation forcée constituait une forme de torture prohibée par l'article $3 \mathrm{CEDH}$ quand elle impliquait d'entraver un prisonnier et de lui insérer de force une sonde de gavage (affaire Nevmerjitski v. Ukraine, $n^{\circ}$ 54825/00, 2005: utilisation de menottes, d'un écarteur buccal et d'un tube en caoutchouc spécial inséré dans l'œsophage; affaire Ciorap v. Moldova, $\mathrm{n}^{\circ}$ 12066/02, 2007).

5 Guillod O, Sprumont D, op.cit., [Rz 30] Le Tribunal fédéral a également omis de signaler que dans une affaire récente, la Cour européenne des droits de l'homme a jugé que le décès d'un détenu causé par sa grève de la faim ne constituait pas une violation des droits humains, dans la mesure où le détenu avait eu accès en prison aux mêmes soins qu'à l'extérieur. La Cour a estimé «que les autorités ne peuvent pas être critiquées non plus d'avoir accepté le refus clair de toute intervention de M. Horoz, alors que son état de santé menaçait sa vie» (affaire Horoz v. Turkey, nº 1639/03, 2009).

halb des Gefängnisses wird Ärzten bisweilen die Berufung auf ihr Gewissen verwehrt, wenn es «eine schwere und unmittelbare Gefahr für die Gesundheit» abzuwenden gilt [19].

\section{Unabhängigkeit des Arztes}

Auch das Argument der zwingenden Unabhängigkeit des Arztes führt zu keiner grundlegend anderen Einschätzung: Nationale und internationale Empfehlungen, allen voran die erwähnten Richtlinien der SAMW, statuieren eine möglichst weitgehende Unabhängigkeit des Arztes von vorgesetzten Behörden. In Bezug auf die medizinisch-fachliche Unabhängigkeit bestehen hierfür gute Gründe, denn die ärztliche Tätigkeit steht in einem systembedingten Spannungsfeld: Der Arzt ist sowohl seinem Patienten, seiner Profession wie auch der Gefängnisleitung gegenüber zu Loyalität verpflichtet. Dieser Konflikt lässt sich weitgehend lösen, indem ihm in Bezug auf medizinische Fragen umfassende Unabhängigkeit von der Verwaltung zugestanden wird. Die Frage des «Ob» einer Zwangsernährung tangiert jedoch - sofern medizinisch keine andere Massnahme zur Lebensrettung mehr zur Verfügung steht - diese Unabhängigkeit nur sehr am Rande und fällt in die Entscheidkompetenz der Justizverwaltung [20].

Dass die auf Vertrauen basierende (Gefängnis-) Arzt-Patienten-Beziehung durch die Androhung und schliesslich durch die Durchführung einer Zwangsmassnahme strapaziert werden kann, steht ausser Frage. Während das Verhältnis zwischen dem Gefangenen und den Vollzugsorganen offensichtlich schwer gestört ist, ist der Arzt möglicherweise eine der letzten Kontaktpersonen, die den dringend notwendigen kommunikativen Zugang zum Streikenden aufrechterhalten kann. Diese Störung des Vertrauensverhältnisses kann allein jedoch kein Argument gegen staatliches Zwangshandeln sein. Der Blick des Staates darf sich - im Gegensatz zu dem eines Privatarztes - nicht auf die einzelne Staat-Bürger-Beziehung beschränken; er hat stets das Gesamte im Auge zu behalten. Augenmass zu bewahren und gleichzeitig das zwingende Recht durchzusetzen, notfalls gegen den Willen von Bürgerinnen und Bürgern, gehört zu den täglichen Herausforderungen des Staates und sichert letztlich das Vertrauen der Gemeinschaft in dessen ordnungsgemässes Funktionieren. Dieses Vertrauen geniesst bisweilen höhere Priorität als das (selbstgerecht ausgelegte) Selbstbestimmungsrecht eines rechtskräftig verurteilten Straftäters.

\section{Zusammenfassung}

Der Königsweg aus einem Hungerstreik führt über den freiwilligen Streikabbruch. Scheitert er, und ist das Leben des Gefangenen nicht anders zu retten, muss der Staat als Ultima ratio zur (einmaligen) Zwangsernährung schreiten. Dies verlangt die im Gefängnis erhöhte staatliche Fürsorgepflicht, die den Schutz des Lebens über den Schutz des selbstbestimmten Sterbens stellt. Dies umso mehr, als Zweifel am ehedem geäusserten Sterbewillen bestehen.

Der Gefängnisarzt erfüllt eine amtliche Tätigkeit. Er ist folglich an das staatliche Recht, an hierarchische Dienstbefehle sowie an Gerichtsentscheide gebunden. Standesregeln sind für ihn nur von subsidiärer Bedeutung. Verweigert der Amtsarzt den dienstlichen Gehorsam, drohen ihm straf- und disziplinarrechtliche Konsequenzen. Dagegen hilft ihm weder der Rekurs auf seine Gewissensfreiheit noch auf seine fachliche Autonomie. Ärztliches Gewissen und ärztliche Autonomie müssen insoweit der Zweckrationalität des Strafvollzugs weichen. 


\section{Literatur}

1 Gravier B, Wolff H, Sprumont D, Ricou B, Kind C, Eytan A et al. Ein Hungerstreik ist eine Protesthandlung. Schweiz Ärztezeitung. 2010;91(39):1521-25.

2 Als Hungerstreik gilt eine im Vollbesitz psychischer Gesundheit begonnene, anhaltende, völlige oder partielle Nahrungsverweigerung mit der Absicht, gegen Umstände oder Massnahmen zu protestieren oder etwas einzufordern, das mit anderen Mitteln nicht erreichbar scheint (vgl. Pont J. Ethische Überlegungen zu Hungerstreik und Zwangsernährung. In: Keppler, Stöver (Hrsg.). Gefängnismedizin. Stuttgart/New York: Thieme; 2009. S. 253.

3 So Riekenbrauck W. Hungerstreik und Zwangsernährung - Erfahrungen aus der Praxis. In: Keppler, Stöver (Hrsg.). Gefängnismedizin. Stuttgart/New York: Thieme; 2009. S. 262.

4 Bundesgerichtsentscheid vom 26.8.2010 («Rappaz», 6B_599/2010, zur Publikation vorgesehen), E. 6.1.1 (mit Hinweisen auf die Rechtsprechung des EGMR); siehe ferner Frowein J, Peukert W. EMRK-Kommentar, 3. Aufl., Kehl am Rhein; 2009, Art. 3 Rz. 7.

5 Vgl. Art. 61 des bernischen Gesetzes über den Straf- und Massnahmenvollzug vom 25.6.2003 (SMVG, BSG 341.1).

6 Fall Rappaz (Fn. 4), E. 6.3.1. - Entgegen der von Gravier et al. [1], S. 1524, geäusserten Ansicht kann die polizeiliche Generalklausel nicht nur bei Drittgefährdung (z.B. terroristischen Anschläge, Epidemienbekämpfung), sondern auch in Fällen von Selbstgefährdung zur Anwendung gelangen.

7 Vgl. Motion Nationalrat Schmidt vom 28.9.2010 («Hungerstreik im Strafvollzug und in der Ausschaffungshaft», 10.3702) mit ablehnender Stellungnahme des Bundesrats (im Plenum noch nicht behandelt).

8 Für weitere Beispiele vgl. Riekenbrauck [3] S. 261.

9 Anders aber Gravier et al. [1] S. 1522.

10 Gemäss Pont [2] S. 254 verliert der Streikende spätestens ab dem 40. Tag die Urteilsfähigkeit, während mit dem Tod bei totalem Fasten spätestens ab dem 75. Tag zu rechnen ist.

11 In diesem Sinn auch Guilbert P, Sebo P, Elger B, Bertrand D. Jeûne de protestation. In: Bertrand, Niveau (Hrsg.). Médecine santé et prison. ChêneBourg; 2006. S. 371.

12 So auch Gravier et al. [1] S. 1523.

13 Vgl. Gravier et al. [1] S. 1525: «Es ist unabdingbar, den Raum der Entscheidungsfindung und -hilfe von gerichtlichen Verfügungen möglichst frei zu halten. Es geht um die Grundfesten der Medizin.» Ferner Krähenmann A, Schweizer A, Tschumi T. Hungerstreik im Strafvollzug. In: Jusletter 10.1.11, Rz. 38. Zur Ambivalenz der SAMW-Richtlinien, die zuweilen auch reine Standesinteressen unter dem Mantel der Ethik kaschierten, vgl. Guillod O. Recht, Ethik und Medizin: Harmonie oder Dissonanzen. In: Bondolfi, Müller (Hrsg.). Medizinische Ethik im ärztlichen Alltag. Basel, Bern; 1999. S. 79.

14 Vgl. auch Fall Rappaz [4], E. 6.2.2 a.E.

15 Fall Rappaz [4] E. 6.3.3.

$16 \mathrm{Zu}$ denken ist dabei an unterlassene Nothilfe (Art. 128 StGB), Ungehorsam gegen amtliche Verfügungen (Art. 292 StGB) und - im Fall des Hinscheidens - ein Strafverfahren wegen vorsätzlicher Tötung durch Unterlassen (Art. 111 StGB). Zu letzterem vgl. auch Riklin F, Zwangsmassnahmen im Bereich der Gesundheitsfürsorge (Verweigerung der Behandlung, Hungerstreik). In: Queloz, Riklin, Senn, de Sinner (Hrsg.). Medizin und Freiheitsentzug. Bern; 2002. S. S. 51.

17 Für alle Guillod O, Sprumont D. Les contradiction du Tribunal fédéral face au jeûne de protestation. In: Jusletter 8.11.10, Rz. 37.

18 So auch Krähenmann, Schweizer, Tschumi [13] Rz. 57.

19 Vgl. Art. 23 Abs. 3 des Gesundheitsgesetzes des Kantons Bern vom 2.12. 1984 (GesG, BSG 811.01).

20 Riklin [16] S. 61 f.; auch Krähenmann, Schweizer, Tschumi [13], Rz. 54. 